\title{
NeuroImage
}

\section{A voxel-based approach to gray matter asymmetries}

\author{
E. Luders, ${ }^{\mathrm{a}, \mathrm{b}}$ C. Gaser, ${ }^{\mathrm{c}}$ L. Jancke, ${ }^{\mathrm{d}}$ and G. Schlaug ${ }^{\mathrm{b}, *}$ \\ anstitute of Experimental and General Psychology, University of Magdeburg, Germany \\ ${ }^{\mathrm{b}}$ Department of Neurology, Beth Israel Deaconess Medical Center and Harvard Medical School, 02215, USA \\ ${ }^{\mathrm{c}}$ Department of Psychiatry, University of Jena, Germany \\ ${ }^{\mathrm{d}}$ Department of Neuropsychology, University of Zurich, Switzerland
}

Received 3 September 2003; revised 20 January 2004; accepted 21 January 2004

Available online 2 April 2004

\begin{abstract}
Voxel-based morphometry (VBM) was used to analyze gray matter (GM) asymmetries in a large sample $(n=60)$ of male and female professional musicians with and without absolute pitch (AP). We chose to examine these particular groups because previous studies using traditional region-of-interest (ROI) analyses have shown differences in hemispheric asymmetry related to $A P$ and gender. Voxel-based methods may have advantages over traditional ROI-based methods since the analysis can be performed across the whole brain with minimal user bias. After determining that the VBM method was sufficiently sensitive for the detection of differences in GM asymmetries between groups, we found that male AP musicians were more leftward lateralized in the anterior region of the planum temporale (PT) than male non-AP musicians. This confirmed the results of previous studies using ROI-based methods that showed an association between PT asymmetry and the AP phenotype. We further observed that male nonAP musicians revealed an increased leftward GM asymmetry in the postcentral gyrus compared to female non-AP musicians, again corroborating results of a previously published study using ROI-based methods. By analyzing hemispheric GM differences across our entire sample, we were able to partially confirm findings of previous studies using traditional morphometric techniques, as well as more recent, voxel-based analyses. In addition, we found some unusually pronounced GM asymmetries in our musician sample not previously detected in subjects unselected for musical training. Since we were able to validate gender- and AP-related brain asymmetries previously described using traditional ROI-based morphometric techniques, the results of our analyses support the use of VBM for examinations of GM asymmetries.
\end{abstract}

(C) 2004 Elsevier Inc. All rights reserved.

Keywords: Brain asymmetry; Planum temporale; Musicians; Gender; Morphometry; Absolute pitch

\section{Introduction}

Traditional postmortem and in vivo morphometric studies have detected some regional brain asymmetries, the most consistent of

* Corresponding author. Music and Neuroimaging Laboratory, Beth Israel Deaconess Medical Center and Harvard Medical School, Palmer 1, 330 Brookline Avenue, Boston, MA 02215. Fax: +1-617-632-8920.

E-mail address: gschlaug@caregroup.harvard.edu (G. Schlaug).

Available online on ScienceDirect (www.sciencedirect.com.) which include the leftward occipital, and rightward frontal extensions of the brain (Chiu and Damasio, 1980; LeMay, 1977), and the leftward asymmetry of the planum temporale (PT) (Geschwind and Levitsky, 1968; Shapleske et al., 1999; Steinmetz, 1996; Wada et al., 1975). Given that the PT is a cerebral structure located within the posterior superior bank of the temporal lobe, its asymmetry is strongly related to hemispheric differences in the trajectory of the Sylvian fissure running steeper in the right hemisphere while it extends further back in the left (Loftus et al., 1993; Ratcliff et al., 1980). In addition, differences in asymmetry between groups defined by gender, handedness, or according to their functional characteristics were reported (see Jancke and Steinmetz, 2003; Toga and Thompson, 2003 for a summary of this research). These studies have typically defined particular region-of-interests (ROIs) and measured their size and hemispheric asymmetry using traditional morphometric techniques.

In contrast to ROI-based methods, a fully automated voxel-byvoxel analysis technique allows the examination of cerebral asymmetries across the entire brain while it avoids the subjectivity of ROI approaches. Voxel-based morphometry (VBM) has been described in detail (Ashburner and Friston, 2000; Good et al., 2001a,b), and validated with structural ROI analyses and functional studies (Good et al., 2002; Krams et al., 1999; Sowell et al., 1999; Tisserand et al., 2002; Woermann et al., 1999). With regard to hemispheric differences within a study group, VBM was shown to replicate previous findings of frontal and occipital petalias, asymmetries of posterior language areas including the PT, the cingulate sulcus, and caudate nucleus in normal adult human brains (Good et al., 2001a; Watkins et al., 2001). However, studies comparing gray matter (GM) asymmetry between two groups have yielded conflicting results. While Good et al. (2001a) confirmed increased leftward asymmetry within Heschl's gyrus (HG) and the PT in males compared to females, Watkins et al. (2001) did not find any gender differences in the degree of cerebral asymmetry. These discrepant results raise the question whether the VBM technique is sufficiently sensitive for detecting differences in cerebral asymmetries. Consequently, this study's first goal was to verify whether VBM is an alternative to traditional ROI-based methods for detecting GM asymmetry differences between different groups. Conventional ROI-based morphometry has already been shown to yield regional asymmetry differences between 
groups (e.g., Keenan et al., 2001; Kulynych et al., 1994; Schlaug et al., 1995b; Zatorre et al., 1998). In order to contrast these two techniques, we chose to compare manually obtained regional asymmetry scores for the PT using traditional morphometric techniques with asymmetry scores obtained automatically on a voxel-by-voxel basis across the entire brain within the same subjects.

The second goal was to compare GM asymmetries between musicians with absolute pitch (AP) and musicians without AP. As observed in traditional ROI-based analyses (Keenan et al., 2001; Schlaug et al., 1995b), AP musicians have a more pronounced leftward PT asymmetry than non-AP musicians or nonmusicians. Similarly, Zatorre et al. (1998) detected a greater leftward PT asymmetry when AP musicians were compared to a large control sample, although no differences were found when comparing smaller samples. Finally, we wanted to determine whether there is a gender effect or an interaction between gender and AP with respect to GM asymmetry. Amunts et al. (2000) showed that male right-handers have a significantly deeper central sulcus on the left hemisphere than on the right, whereas no interhemispheric asymmetry was found in female right-handers. Furthermore, a genderdependent PT asymmetry with males having a significantly larger left versus right PT, and females showing either diminished or no PT asymmetry, was reported by Kulynych et al. (1994) based on the analysis of a predefined brain region and by Good et al. (2001a) based on a voxel-by-voxel analysis. Other studies, however, did not find a gender effect in PT asymmetry (Foundas et al., 1999; Watkins et al., 2001).

\section{Materials and methods}

\section{Subjects}

The study group consisted of 60 healthy, right-handed musicians (40 non-AP musicians: 20 females/20 males, and 20 AP musicians: 10 females/10 males) who were selected from a database of high resolution anatomical MR images. The sample was matched according to gender (30 women, 30 men) and had a mean age of 24.7 years $( \pm 4.7)$. All musicians were classically trained professionals (mainly keyboard and string players) who were either performing artists, teachers, or students at local music schools. The mean age for commencement of music training was 6.4 years $( \pm 2.6)$. Right-handedness was confirmed in all subjects with standardized tests (Annett, 1992; Jancke et al., 1997). AP was verified using well-established tests (Keenan et al., 2001; Ward and Burns, 1982; Zatorre et al., 1998). A detailed description of the AP testing procedure is provided in Keenan et al. (2001). The study was approved by the Institutional Review Board of Beth Israel Deaconess Medical Center Boston, MA.

\section{MRI acquisition and data analysis}

High-resolution anatomical images of the whole brain were obtained on a 1.5 T Siemens Vision whole-body scanner (Erlangen, Germany) by using a strongly T1-weighted gradient echo pulse sequence (MP-RAGE) with parameters described in detail by Keenan et al. (2001). Image analysis was performed on a PC using MATLAB 5.3 (Mathworks Inc., Natick, MA, USA; http://www. mathworks.com/products/matlab) and SPM99 software (Wellcome Department of Cognitive Neurology, London; http:/www.fil.ion. ucl.ac.uk/spm/). Subsequent statistical analyses were performed using SPSS 10.0 (SPSS Inc. 1989-99; http://www.spss.com).

\section{Image preprocessing}

\section{Creating templates: whole-brain and symmetrical GM template}

A whole-brain template was created from $56 \mathrm{~T} 1$-weighted images (36 males, 20 females) chosen from right-handed subjects scanned in our institution over the past 5 years. These 56 images were stereotaxically transformed applying a 12-parameter affine transformation to match the Montreal Neurology Institute (MNI) 305 template. After the normalizing step, one average image was created and smoothed with an 8-mm FWHM isotropic Gaussian kernel.

The 56 normalized, T1-weighted images were segmented and extracted, then flipped vertically in the midsagittal plane $(x=0)$ to create a symmetrical GM template. Finally, one average image from all GM images (normal and flipped) was created and smoothed with an 8-mm FWHM isotropic Gaussian kernel. In addition, we created symmetrical a priori maps by averaging the GM, white (WM), and cerebrospinal fluid (CSF) a priori maps provided with the SPM99 package, with their flipped counterparts. The symmetrical GM template and a priori maps were used in the subsequent spatial normalization and segmentation procedures described below.

\section{Verifying VBM sensitivity in detecting GM asymmetry differences}

A data set of 52 brains that partly overlapped with the sample of 60 musicians used in the current analysis was available with a wide range of PT asymmetry scores. These scores were obtained by manually determining the PT surface area using splines to approximate the contour of the PT on sagittal slices and forming a triangular mesh between contours (for details, see Keenan et al., 2001). This set of brains was chosen from a larger group (Keenan et al., 2001) including only MR data sets that were obtained on the same MR scanner. In order to determine whether VBM is sufficiently sensitive to detect GM asymmetry differences, we calculated the correlation between the 52 PT asymmetry scores obtained manually and the 52 asymmetry indices obtained automatically on a voxel-by-voxel basis across the whole brain. The asymmetry index of the PT's surface area was calculated using the formula (Right - Left) $/ 0.5$ (Right + Left). The set of 52 subjects included AP musicians $(n=25)$, non-AP musicians $(n=10)$, and nonmusicians $(n=17)$, thus representing individuals with a large range of PT asymmetry indices. The image preprocessing steps for manual segmentation (e.g., data acquisition, PT definition, PT measuring, and PT surface calculation) are described in detail by Keenan et al. (2001). Voxel-by-voxel GM asymmetry scores were determined as described below.

\section{Applying VBM to examine GM asymmetry}

Each T1-weighted, MR data set from our study group $(n=60)$ was linearly transformed into MNI standard space using our wholebrain template. We then segmented the normalized images into GM, WM, CSF, and other nonbrain components by applying the SPM99 segmentation algorithm that also includes a correction for nonuniformity in image signal intensities (Ashburner and Friston, 2000). Applying an additional series of fully automated, morphological operations, we removed scalp tissue, skull, and dural venous sinuses from the GM volumes (Good et al., 2001b). The extracted GM volumes were then used to estimate the spatial normalization parameters by matching them to our self-created symmetrical GM template using linear (12 parameter affine transformation) and nonlinear components $(7 \times 8 \times 7$ basis functions $)$. These trans- 


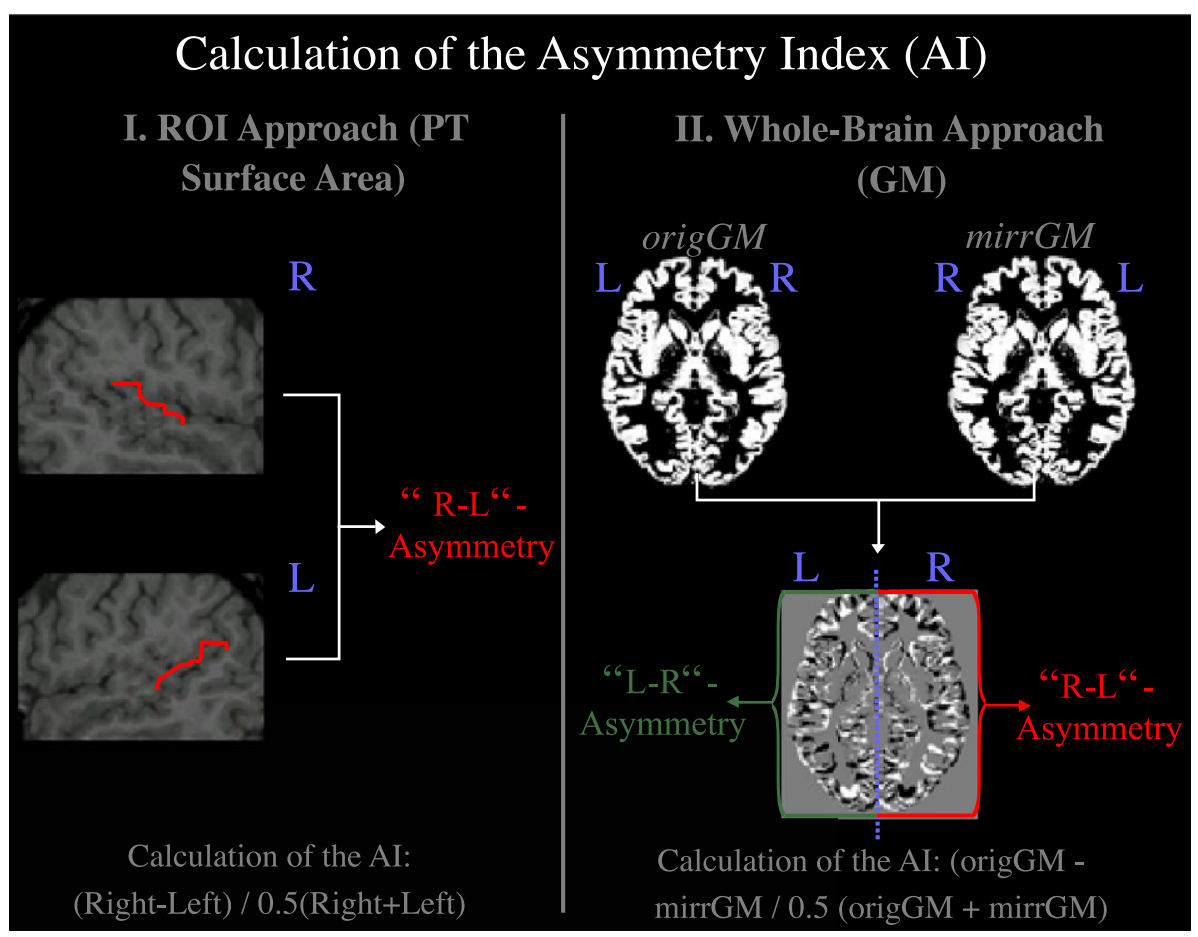

Fig. 1. Calculation of asymmetry indices (AIs). The AIs for the PT surface area were calculated by subtracting left hemispheric values from the right resulting in "right-minus-left"-asymmetries. Whole-brain AIs were calculated by subtracting the mirrored images from the original images. Thus, the resulting voxelwise AIs in the left hemisphere represent "left-minus-right"-asymmetries, while AIs in the right hemisphere represent "right-minus-left"-asymmetries. Therefore, positive correlations between AIs obtained with the two different approaches will arise in the right hemisphere (while negative correlations will arise in the left hemisphere).

formations were used to spatially normalize the original T1-weighted images. After reslicing image volumes into isotropic voxels (1 $\mathrm{mm}^{3}$ ), we performed another segmentation procedure using our modified symmetrical a priori maps. After the unconnected, nonbrain voxels were removed, the resulting GM images were extracted (Good et al., 2001b).

In order to examine asymmetries, we generated a new set of GM images by calculating a GM difference image (DI) for each subject by flipping the normalized and extracted original GM images (origGM) vertically in the midsagittal plane $(x=0)$. This resulted in mirrored GM images (mirrGM) to which we applied the following formula:

$\mathrm{DI}=($ origGM $-\operatorname{mirrGM}) / 0.5($ origGM + mirrGM $)$

These difference images represent the GM differences between the two hemispheres for each voxel. Positive voxel values on the right side of the image indicate that the right hemisphere had higher GM intensity value than the left; negative voxel value on the right side of the image indicate that the left hemisphere had higher GM intensity values than the right. Images were then smoothed using a 12-mm FWHM isotropic Gaussian kernel.

\section{Statistical analyses}

\section{$V B M$ sensitivity}

Asymmetry scores obtained manually for PT surface area were correlated with asymmetry scores obtained automatically on a voxel-by-voxel basis across the entire brain. Using conventional morphometry, the measurement of the PT surface is influenced by the length and convolution of the PT region. Based on the assumption that the latter is related to the regional GM volume, we expected to find positive correlations in the region of the PT if $\mathrm{VBM}$ is as sensitive as traditional ROI methods in detecting
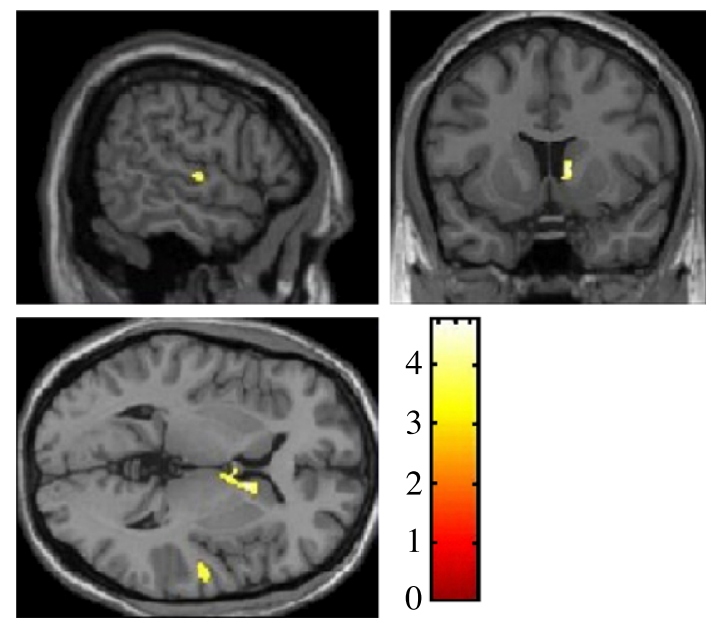

Fig. 2. Positive correlations between manually (PT region) and automatically (whole-brain) obtained asymmetry scores. By applying a linear regression, we found a positive correlation in the region of the PT and the caudate nucleus. Since we had a clearly defined regional hypothesis, the statistical map was thresholded at $P \leq 0.001$ and not corrected for multiple comparisons. Only clusters thresholded with the number of expected voxels (spatial extent threshold of $n=48$ ) are shown. As a result of calculating the asymmetry index by subtracting the values of the left hemisphere from the right (ROI approach), and subtracting the mirrored images from the original images (whole-brain approach), all positive correlations arise exclusively in the right hemisphere. The color bar represents the $t$ score. 
between-group differences in cerebral asymmetries. Our preexisting hypothesis predicted correlations in the PT region; therefore, the statistical map was thresholded at $P \leq 0.001$ and not corrected for multiple comparisons. Only clusters thresholded with the number of expected voxels (spatial extent threshold) calculated according to the theory of Gaussian random fields are reported. Due to the fact that we calculated the whole-brain asymmetry index by subtracting the flipped images from the original images, the resulting voxelwise asymmetry indices in the left hemisphere represent "left-minusright"-asymmetries, while asymmetry indices in the right hemisphere represent "right-minus-left"-asymmetries (Fig. 1). As described above, the manually obtained asymmetry indices for the PT region were obtained by subtracting the left from the right hemisphere ("right-minus-left"-asymmetry). Therefore, positive correlations between asymmetry indices obtained with the two different approaches should arise in the right PT region.

\section{GM asymmetry differences between groups defined by gender and $A P$}

The smoothed GM difference images of our study group $(n=60)$ were analyzed using a one-way analysis of variance (ANOVA). The design matrix employing the general linear model represented the GM difference images of female AP musicians $(n=10)$, female non-AP musicians $(n=20)$, male AP musicians $(n=10)$, and male non-AP musicians $(n=20)$. We created different contrasts comparing GM asymmetries between males and females and between AP musicians and non-AP musicians. In addition, we investigated the possibility of interaction effects between AP and gender in association with GM asymmetries. Significance levels for the $t$ statistics were set at $P<0.05$, corrected for multiple comparisons across the brain volume (Benjamini and Hochberg, 1995). Only clusters thresholded with the number of expected voxels (spatial extent threshold), calculated according to the theory of Gaussian random fields, are reported.

\section{GM asymmetry across the entire sample of musicians}

The smoothed GM difference images of all subjects $(n=60)$ were analyzed using a one-sample $t$ test. To examine the effects of $\mathrm{AP}$ and gender on a specific cerebral region previously described as showing asymmetry differences between AP and non-AP musicians, and between males and females, we conducted follow-up analyses. Here we focused on the superior temporal gyrus, and assessed GM asymmetry in groups defined by gender (males, females) and/or musician status (AP musician, non-AP musician). Significance was assigned to the resulting $t$ fields using the theory of Gaussian random fields. The statistical map was thresholded at $P \leq$ 0.05 , corrected for multiple comparisons across the volume (Benjamini and Hochberg, 1995). Again, only clusters thresholded with the number of expected voxels (spatial extent threshold) calculated according to the theory of Gaussian random fields are reported.

All preprocessing steps and statistical analyses described in the Materials and methods are summarized in Table 1.

\section{Results}

Correlation of asymmetry indices obtained with different approaches

The linear regression analysis between asymmetry indices obtained automatically on a voxel-by-voxel basis across the entire brain, and PT surface area asymmetry indices determined manually, revealed a significant positive correlation in the anterior PT region (Fig. 2). A further significant positive

Table 1

Summary of preprocessing steps and statistical analyses

\begin{tabular}{|c|c|c|c|c|}
\hline & $\begin{array}{l}\text { Creating a template: } \\
\text { (a) whole-brain template, } \\
\text { (b) symmetrical GM template }\end{array}$ & $\begin{array}{l}\text { Verifying VBM sensitivity } \\
\text { in detecting GM asymmetry } \\
\text { differences }\end{array}$ & $\begin{array}{l}\text { Examining GM asymmetry } \\
\text { differences between groups } \\
\text { defined by gender and AP }\end{array}$ & $\begin{array}{l}\text { Examining GM asymmetry } \\
\text { across the entire sample } \\
\text { of musicians }\end{array}$ \\
\hline Subjects & $n=56$ (36 males, 20 females $)$ & $\begin{array}{l}n=52(25 \text { AP musicians, } \\
10 \text { non-AP musicians, } \\
17 \text { nonmusicians })\end{array}$ & \multicolumn{2}{|c|}{$\begin{array}{l}n=60 \text { ( } 30 \text { males, } 30 \text { females; } 20 \text { AP musicians, } \\
40 \text { non-AP musicians) }\end{array}$} \\
\hline Preprocessing & $\begin{array}{l}\text { whole-brain template: } \\
\text { (1) 12-parameter affine transformation } \\
\text { (MNI305 template) } \\
\text { (2) averaging the } 56 \text { whole-brain images } \\
\text { (3) smoothing the average whole-brain } \\
\text { image ( } 8 \mathrm{~mm} \text { ) } \\
\text { symmetrical GM template: } \\
\text { (1) } 12 \text {-parameter affine transformation } \\
\text { (MNI305 template) } \\
\text { (2) segmentation (SPM99 a priori maps) } \\
\text { (3) flipping the GM images } \\
\text { (vertically, } x=0 \text { ) } \\
\text { (4) averaging the } 56 \text { non-flipped } \\
\text { and } 56 \text { flipped GM images } \\
\text { (5) smoothing the average GM image }\end{array}$ & $\begin{array}{l}\text { ROI asymmetry measures: } \\
\text { (1) Manually drawing splines to } \\
\text { represent the PT surface } \\
\text { (2) calculating the asymmetry } \\
\text { index (AI) of the PT surface: } \\
\text { AI = (R - L) } / 0.5(\mathrm{R}+\mathrm{L}) \\
\text { VBM asymmetry measures: } \\
\text { (1) } 12 \text {-parameter affine transforma } \\
\text { (2) segmentation (SPM99 a priori } \\
\text { (3) parameter estimation for spatia } \\
\text { (4) spatial normalization of the or } \\
\text { (5) segmentation (symmetrical a p } \\
\text { (6) flipping the GM images (verti } \\
\text { (7) calculating the difference GM } \\
\text { (8) smoothing the difference GM }\end{array}$ & $\begin{array}{l}\text { ion (whole-brain template) } \\
\text { maps) } \\
\text { normalization (GM images, } \\
\text { ginal images (linear + non-lin } \\
\text { iori maps) } \\
\text { ally, } x=0) \\
\text { mages: } \mathrm{DI}=(\text { origGM }- \text { mirrG } \\
\text { mages }(12 \mathrm{~mm})\end{array}$ & $\begin{array}{l}\text { mmetrical GM template) } \\
\text { r components) } \\
\text { 1) } 0.5 \text { (origGM }+ \text { mirrGM) }\end{array}$ \\
\hline Statistics & & $\begin{array}{l}\text { correlation between ROI } \\
\text { asymmetry scores for the PT } \\
\text { region and VBM asymmetry } \\
\text { scores for the entire brain } \\
(P \leq 0.001, \text { not corrected })\end{array}$ & $\begin{array}{l}\text { ANOVA in a two } \\
\text { (males, females) by two } \\
\text { (AP musicians, non-AP } \\
\text { musicians) design } \\
(P \leq 0.05, \text { corrected) }\end{array}$ & $\begin{array}{l}\text { one-sample } t \text { test } \\
(P \leq 0.05, \text { corrected })\end{array}$ \\
\hline
\end{tabular}




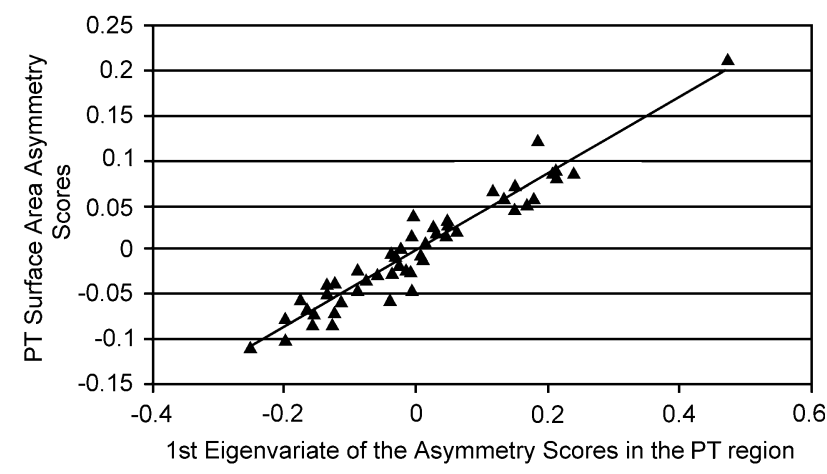

Fig. 3. Relationships between asymmetry scores obtained with different approaches. Correlating the first eigenvariates of automatically obtained asymmetry scores in a defined set of PT region voxels with manually obtained asymmetry scores for the PT surface areas revealed a highly significant, positive correlation $(r=0.96 ; P \leq 0.01)$.

correlation was observed in the head of the caudate nucleus (Fig. 2).

In order to provide further evidence supporting a positive relationship between both sets of asymmetry scores, we extracted the first eigenvariate of automatically obtained GM asymmetry scores for a sphere with a radius of $8 \mathrm{~mm}$ centered around the maximum in the PT region. The first eigenvariate of this cluster accounted for most of the variance in the automatically obtained GM asymmetry scores in this defined set of voxels. A correlation of $r=0.96(P=0.01)$ was found when the 52 eigenvariates were correlated with the 52 manually obtained asymmetry scores of the PT surface area (Fig. 3).

\section{Between-group GM asymmetry differences}

By comparing GM asymmetries between groups, we observed that male AP musicians showed increased leftward asymmetries in the anterior region of the PT compared to male non-AP musicians (Fig. 4a). Furthermore, male non-AP musicians revealed an increased leftward GM asymmetry in the postcentral gyrus compared to female non-AP musicians (Fig. 4b). None of the other comparisons taking AP, gender, and the interaction between the two into account (all females vs. all males, all AP musicians vs. all non-AP musicians, female AP musicians vs. female non-AP musicians, etc.) resulted in statistically significant suprathreshold voxels.

\section{GM asymmetries in musicians-whole brain}

When hemispheric GM differences were analyzed within the entire group of 60 professional musicians, the analysis revealed extensive rightward GM asymmetries $(\mathrm{R}>\mathrm{L})$ in the frontal and prefrontal lobe, mesial portions of the superior temporal lobe, posterior parts of the inferior and medial temporal gyrus, and temporal pole. In addition, more focal rightward GM asymmetries were detected in the superior parts of the pre- and postcentral gyrus, the inferior and superior parietal lobe, the cuneus, cingulate, and lingual gyrus, as well as in the orbital and medial occipital gyrus (Fig. 5, upper row). We also observed a dominant rightward GM asymmetry in most of the cerebellum with some small foci of leftward GM asymmetry in the dorsal, posterior, and anterior regions of the cerebellum. Leftward GM asymmetries $(\mathrm{L}>\mathrm{R})$ were also detected in the superior temporal gyrus including $\mathrm{HG}$, and in the anterior portion of the PT. Additional clusters of leftward GM asymmetry were demonstrated in the inferior parts of the preand postcentral gyrus, the mesial frontal lobe, thalamus and caudate nucleus, and occipital pole (Fig. 5, lower row).

\section{GM asymmetries defined by gender and AP-superior temporal} gyrus

AP musicians (males and females combined) showed a significant leftward GM asymmetry in the superior temporal gyrus including HG and anterior portion of the PT. However, when male and female APs were analyzed separately, a clear leftward GM asymmetry in the anterior PT region was only seen in male AP musicians. Female AP musicians, on the other hand, demonstrated hemispheric GM differences anterior to the PT, in the HG (Fig. 6, upper row). Similar results were found in the group of female nonAP musicians who showed hemispheric GM differences slightly

Fig. 4. Brain regions with significant differences of GM asymmetries. The voxel-by-voxel comparison of GM asymmetries between groups revealed two significant differences: (a) the left anterior portion of the PT in male AP musicians was more lateralized than in male non-AP musicians, and (b) the left postcentral gyrus in male non-AP musicians was more lateralized than in female non-AP musicians $(P<0.05$, corrected for multiple comparisons, spatial extent threshold, $n=16$ ). The color bar represents the $t$ score.

Fig. 5. Brain regions with significant hemispheric GM differences in a sample of 60 professional musicians. Rightward GM asymmetries (R $>$ L) were seen in the frontal and prefrontal lobe (a, blue arrows), mesial portions of the superior temporal lobe (b, blue arrow), posterior parts of the inferior and medial temporal gyrus (a, green arrows), superior parts of the pre- and postcentral gyrus (a, yellow arrows), inferior and superior parietal lobe (a, cyan arrows), cuneus and lingual gyrus (c, blue arrows), cingulate gyrus (d, blue arrow), medial occipital gyrus (a, purple arrow), and in some regions of the cerebellum (c, green arrow). Leftward GM asymmetries ( $\mathrm{L}>\mathrm{R}$ ) were seen in the superior temporal gyrus including HG and anterior portion of the PT (e, blue arrow), inferior parts of the pre- and postcentral gyrus (e, yellow arrows), mesial frontal lobe (g, blue arrow), thalamus and caudate nucleus (h, blue arrows), occipital pole (f, blue arrow), and in dorsal, as well as most posterior and anterior portions of the cerebellum (e and f, green arrows). Only clusters corrected for multiple comparisons $(P<$ 0.05 ) and thresholded with the number of expected voxels - spatial extent threshold of 163 voxels per cluster-are shown here.

Fig. 6. Brain regions with significant leftward GM asymmetries in the superior temporal gyrus. The combined group of AP musicians (all APs) showed significant leftward GM asymmetries in the superior temporal gyrus including HG and anterior portions of the PT $(P<0.05$, corrected for multiple comparisons). When male and female APs are analyzed separately, a leftward GM asymmetry in the anterior PT was found in the male sample (male APs) with significant differences in the upper-left quadrant of the crosshair (placed in Heschl's sulcus at $x=-53 ; y=-18 ; z=0$, MNI space for all images shown). The female APs demonstrated more anterior hemispheric GM differences (mostly in the upper and lower right quadrant) in HG but not the PT. Similar results were found for the group of female non-AP musicians (female nAPs). In male non-AP musicians (male nAPs), hemispheric GM differences were located even more anterior and inferior (lower right quadrant) compared to the other groups. These findings were clearly reflected in the combined non-AP musician group (all nAPs). The description of significant results is restricted to the superior temporal gyrus in the left hemisphere (LH). 

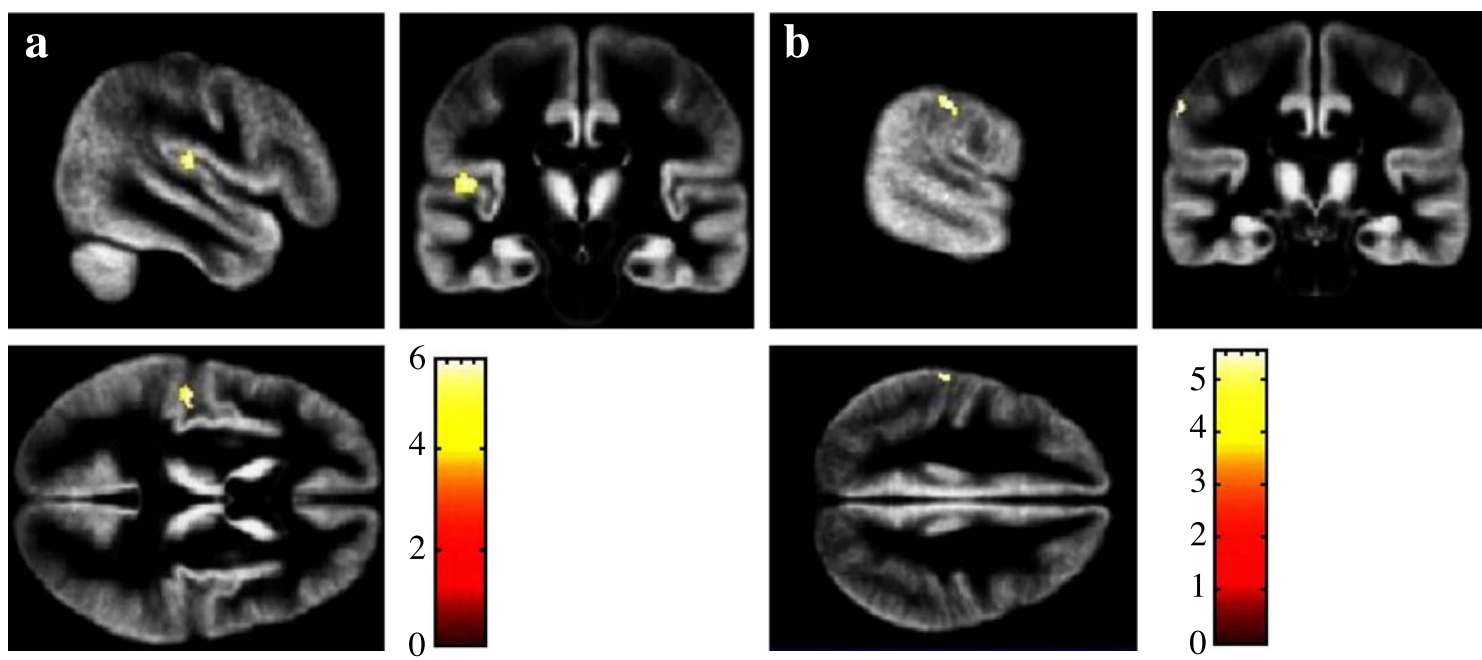

Fig. 4.

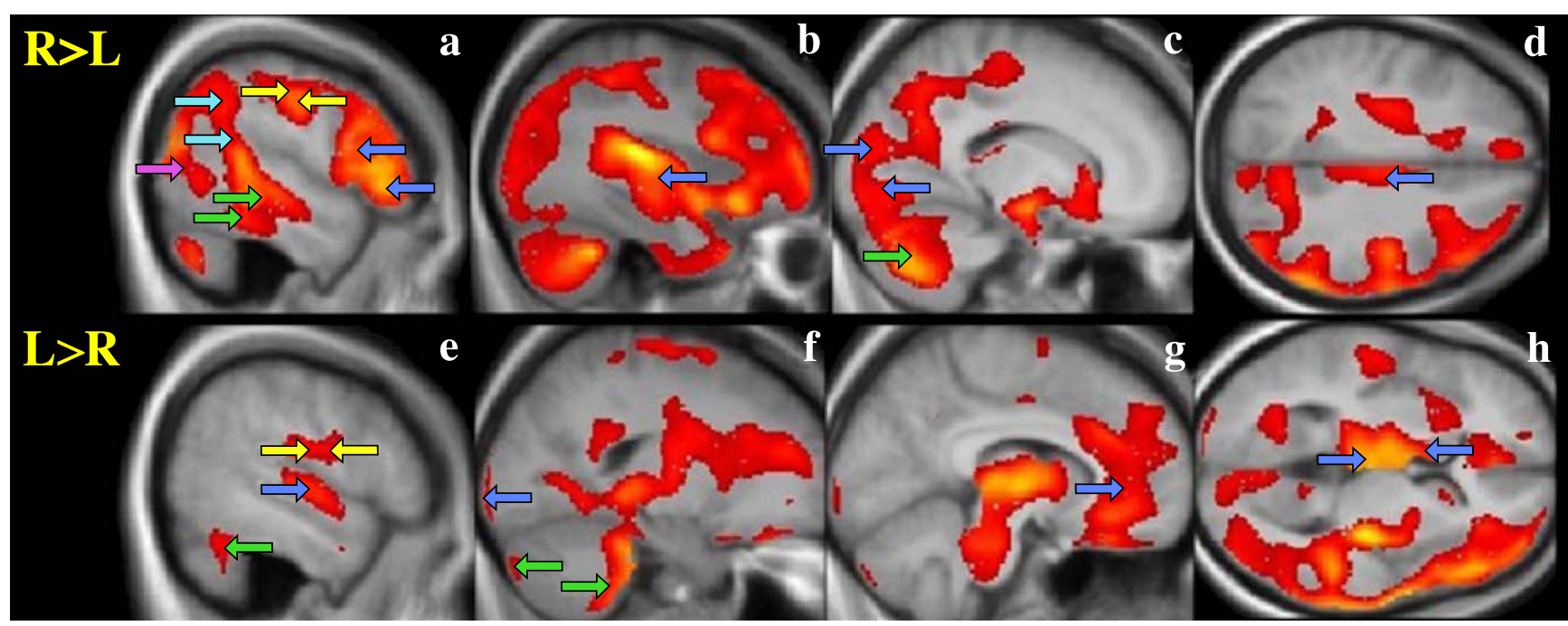

Fig. 5.

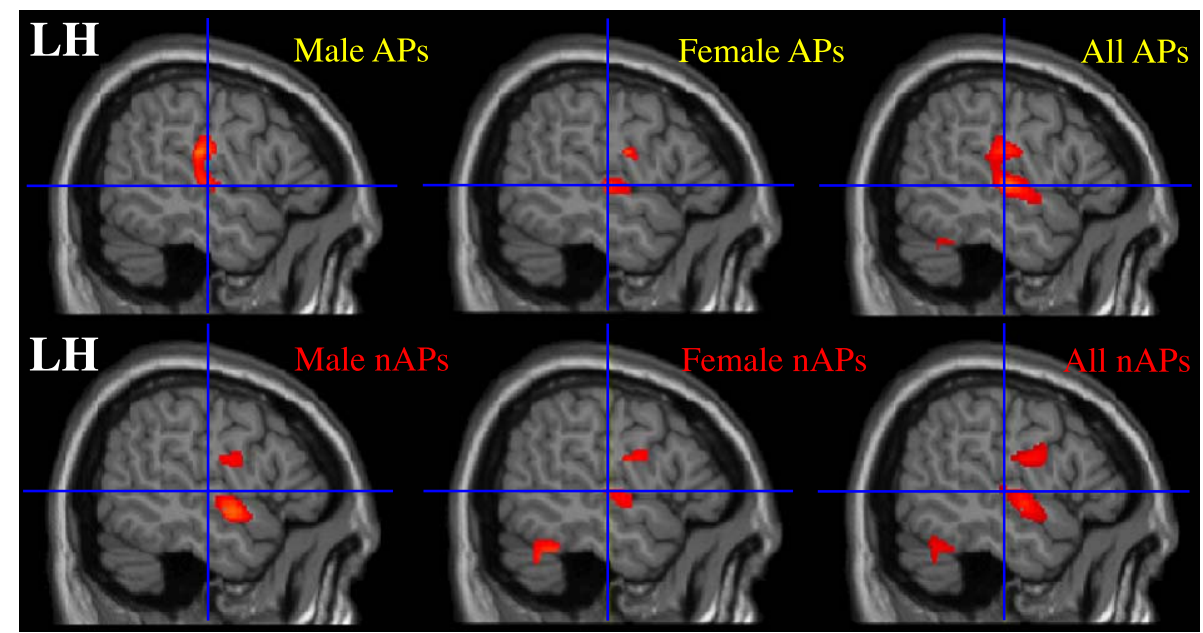

Fig. 6. 
more inferior to the HG than the female AP musicians, whereas in male non-AP musicians, hemispheric GM differences were located anterior to the HG (Fig. 6, lower row).

\section{Discussion}

Previous studies have revealed different results when using VBM methods to detect between-group differences in GM asymmetries. While Good et al. (2001a) detected gender differences in regional GM asymmetries confirming previous results from traditional ROI analyses, Watkins et al. (2001) did not detect a gender effect on hemispheric GM asymmetry. Consequently, our first aim was to test the validity of VBM for detecting GM asymmetries by comparing whole-brain asymmetry indices obtained automatically on a voxel-by-voxel basis, with PT asymmetry indices obtained manually with a conventional ROI-based approach (for details, see Keenan et al., 2001; Schlaug et al., 1995b). The positive correlation between both sets of asymmetry scores mapped to the region of the PT supports the notion that VBM is a sufficiently sensitive method for detecting GM asymmetry differences between groups. There was an additional positive correlation in the caudate nucleus. Although the finding in the caudate nucleus is not relevant for the purpose of validation, it is of great interest considering the animal literature that suggests extensive connections between caudate nucleus and secondary auditory areas as well as auditory association cortex (Baizer et al., 1993; Yeterian and Pandya, 1998). If brain structures localized in different regions (e.g., PT and caudate nucleus) are strongly interconnected, they might resemble each other in terms of asymmetry. This hypothesis, however, warrants further examination in future studies. An alternative explanation is that the manually determined PT asymmetry, which correlated with the GM asymmetry in the anterior PT region and the head of the caudate, reflects hemispheric asymmetries in more than just temporal regions. It might be possible that dorsolateral prefrontal regions, which typically project to the caudate nucleus, are asymmetric. This could lead to a hemispheric asymmetry of the caudate nucleus if the planum temporale is a marker of a greater hemispheric asymmetry. There is some indirect evidence for this, since several studies have shown strong leftward activation of a posterior dorsolateral brain region in pitch experiments in individuals with absolute pitch (Ohnishi et al., 2001; Zatorre et al., 1998); AP musicians typically have an exaggerated leftward PT asymmetry (Keenan et al., 2001).

The second aim of our study was to examine whether GM asymmetries differ between AP musicians and non-AP musicians. We observed a significantly increased leftward GM asymmetry in the anterior PT region in male AP musicians. However, this effect was not observed in comparisons between female samples or when comparing all AP with all non-AP musicians. Nevertheless, the GM asymmetry difference between male AP and male non-AP musicians in the PT region replicates previous studies that described an increased leftward PT asymmetry in AP musicians (Keenan et al., 2001; Schlaug et al., 1995b). The PT was the only region that showed a significant effect when testing across the entire brain space. The current study is the first to find a gender effect in the APmodulated PT asymmetry since previous studies did not investigate whether a gender effect existed (Keenan et al., 2001).

By comparing female and male non-AP musicians, the present study also revealed a significantly increased leftward GM asymmetry in males in a region posterior to the central sulcus. This is of particular interest considering the results of central sulcus measurements from a nonmusician sample. Amunts et al. (2000) demonstrated that right-handed males show a significantly deeper central sulcus on the left hemisphere than on the right, whereas no interhemispheric asymmetry was found in females. A recent functional imaging experiment reported gender-specific perirolandic asymmetries in a tactile discrimination task, where females activated predominantly both premotor cortices, but males showed an asymmetric activation (Sadato et al., 2000). Other studies (e.g., Good et al., 2001a; Hiscock et al., 2001; Kansaku et al., 2000; Kulynych et al., 1994; Lake and Bryden, 1976; Medland et al., 2002) that showed increased anatomical symmetry or decreased functional lateralization in females corroborate the presence of gender-specific cerebral asymmetries. It is also of great interest that both male samples demonstrated GM asymmetries in different brain regions, with male AP musicians showing GM asymmetries in the anterior PT, and male non-AP musicians in the region anterior to HG. In contrast, both female groups (AP and non-AP musicians) showed GM asymmetries in the same location in the region of HG but not in the PT. In agreement with our findings, Kulynych et al. (1994) did not observe any hemispheric differences in the PT in females, despite the differences found in males. These findings suggest that the location and degree of hemispheric GM asymmetries may be strongly determined by gender, AP, and the interaction between the two. A recent replication study from our group revealed a similar finding with males showing a significant musicianship effect in corpus callosum size while females did not show a significant effect (Lee et al., 2003). Similar observations were made when examining the effects of music training intensity and cerebellar volume (Hutchinson et al., 2003). Taken together, prior findings and the results of the present study may suggest a dimorphic organization in the brains of men and women that is detectable in morphometric studies using either voxel-based or traditional ROI-based techniques (Amunts et al., 2000; Foundas et al., 1999; Good et al., 2001a; Nopoulos et al., 2000).

By analyzing hemispheric GM differences within our entire sample of professional musicians $(n=60)$, we confirmed findings of previous ROI-based and VBM studies in normal (nonmusician) samples. These observations include the well-known leftward asymmetry of the superior temporal gyrus including $\mathrm{HG}$ and anterior portion of the PT, as well as the leftward GM asymmetries in the occipital pole corroborating reports of leftward occipital extensions (petalias). Furthermore, we replicated findings of rightward asymmetries in posterior parts of the inferior and medial temporal gyrus (Paus et al., 1996; Watkins et al., 2001) and cingulate gyrus (Good et al., 2001a; Paus et al., 1996; Watkins et al., 2001). We also observed a leftward asymmetry of the caudate nucleus confirming recent VBM findings by Good et al. (2001a). In contrast, other studies reported a rightward asymmetry of the caudate nucleus (Ifthikharuddin et al., 2000; Watkins et al., 2001). Interestingly, we detected rightward GM asymmetries in mesial portions of the superior temporal lobe, in superior parts of the preand postcentral gyrus, the inferior and superior parietal lobe, the cuneus, and the lingual gyrus. These asymmetries have not been observed previously in subjects not selected for musical background. Given that the quality of tissue classification is influenced by the contrast between GM and WM, it is of critical importance for interpreting GM asymmetries that GM and WM contrasts do not differ significantly between homologous regions of both hemispheres. However, it is more likely that GM-WM contrasts are 
different between cortical and subcortical areas, rather than between homologous regions of the two hemispheres. Although some anatomical studies have described subtle cytoarchitectonic differences between right and left superior temporal lobe, these studies have also emphasized that the overall cortical thickness, as well as the overall number of neurons, does not differ much between right and left superior temporal cortex. Thus, it is very unlikely that differences in the GM-WM contrast between right and left hemisphere could explain our findings.

Findings of unusually pronounced rightward GM asymmetries in musicians may be the result of cerebral adaptations to specific environmental challenges, such as learning to play an instrument with particular emphasis on skill development in the nondominant hand (e.g., piano and string instruments). Amunts et al. (1997) found a similar effect in musicians by detecting areal and hemispheric asymmetry differences in an index of precentral gyrus size when instrumentalists were compared with matched nonmusicians. The dominance of changes in the right compared to the left hemisphere as a function of acquiring a musical skill has also been observed in two recent studies. Bangert and Altenmuller (2003) showed the rapid development of a right-hemispheric frontotemporal network in an auditory-motor mapping task. Stewart et al. (2003) demonstrated a right-more-than-left superior parietal activation when learning of musical notation was examined in nonmusicians. In addition, we found some evidence for a rightsided dominance of a pitch memory network involving the inferior parietal lobe when musicians were compared with performancematched nonmusicians (Gaab and Schlaug, 2003). Shahin et al. (2003) also described a right-hemispheric enhancement of the early auditory-evoked potentials when musicians were compared with nonmusicians. The functional significance of the rightward GM asymmetry of the cuneus and lingual gyrus will have to be determined in future studies. Alternatively, these specific hemispheric GM differences in musicians could reflect the innate ability to become a professional musician or acquire exceptional skills like AP. As demonstrated in some ROI-based studies, several brain areas differ in their structure and size when comparing musicians to control subjects (Hutchinson et al., 2003; Lee et al., 2003; Munte et al., 2002; Schlaug, 2001; Schlaug et al., 1995a; Schneider et al., 2002; Zatorre et al., 1998). Additional studies applying VBM have revealed increased GM volumes in motor, auditory, and visualspatial brain regions (Gaser and Schlaug, 2003) as well as in Broca's area (Sluming et al., 2002) when comparing professional musicians with nonmusicians.

Since we were able to validate gender and AP-related brain asymmetries previously described using traditional ROI-based morphometric techniques, the results of our analyses support the use of VBM to analyze GM asymmetries. Our methods and results may help generate hypotheses for future research into group differences of cerebral asymmetries using VBM methods allowing whole-brain analysis with minimal user bias.

\section{Acknowledgments}

This work was supported by the German Academic Exchange Service (DAAD), Deutsche Forschungsgemeinschaft (DFG) JA 737/8-1, a grant from the International Foundation for Music Research and the Recording Academy, and a grant from the National Science Foundation (BCS-0132508). Dr. Schlaug is further supported by a Clinical Investigator Development Award of the Doris Duke Charitable Foundation and a grant from the Dana Clinical Hypothesis Program in Imaging.

\section{References}

Amunts, K., Schlaug, G., Jäncke, L., Steinmetz, H., Schleicher, A., Dabringhaus, A., Zilles, K., 1997. Motor cortex and hand motor skills: structural compliance in the human brain. Hum. Brain Mapp. 5, 206-215.

Amunts, K., Jancke, L., Mohlberg, H., Steinmetz, H., Zilles, K., 2000. Interhemispheric asymmetry of the human motor cortex related to handedness and gender. Neuropsychologia 38, 304-312.

Annett, M., 1992. Parallels between asymmetries of planum temporale and of hand skill. Neuropsychologia 30, 951-962.

Ashburner, J., Friston, K.J., 2000. Voxel-based morphometry-The methods. NeuroImage 11, 805-821.

Baizer, J.S., Desimone, R., Ungerleider, L.G., 1993. Comparison of subcortical connections of inferior temporal and posterior parietal cortex in monkeys. Vis. Neurosci. 10, 59-72.

Bangert, M., Altenmuller, E.O., 2003. Mapping perception to action in piano practice: a longitudinal DC-EEG study. BMC Neurosci. 4, 26.

Benjamini, Y., Hochberg, Y., 1995. Controlling the false discovery rate: a practical and powerful approach to multiple testing. J. R. Stat. Soc., Ser. B 57, 289-300.

Chiu, H.C., Damasio, A.R., 1980. Human cerebral asymmetries evaluated by computed tomography. J. Neurol., Neurosurg. Psychiatry 43, 873-878.

Foundas, A.L., Faulhaber, J.R., Kulynych, J.J., Browning, C.A., Weinberger, D.R., 1999. Hemispheric and sex-linked differences in Sylvian fissure morphology: a quantitative approach using volumetric magnetic resonance imaging. Neuropsychiatry Neuropsychol. Behav. Neurol. 12, $1-10$

Gaab, N., Schlaug, G., 2003. The effect of musicianship on pitch memory in performance matched groups. NeuroReport 14, 2291-2295.

Gaser, C., Schlaug, G., 2003. Brain structures differ between musicians and non-musicians. J. Neurosci. 23, 9240-9245.

Geschwind, N., Levitsky, W., 1968. Human brain: left-right asymmetries in temporal speech region. Science 161, 186-187.

Good, C.D., Johnsrude, I., Ashburner, J., Henson, R.N., Friston, K.J., Frackowiak, R.S., 2001a. Cerebral asymmetry and the effects of sex and handedness on brain structure: a voxel-based morphometric analysis of 465 normal adult human brains. NeuroImage 14, 685-700.

Good, C.D., Johnsrude, I.S., Ashburner, J., Henson, R.N., Friston, K.J., Frackowiak, R.S., 2001b. A voxel-based morphometric study of ageing in 465 normal adult human brains. NeuroImage 14, 21-36.

Good, C.D., Scahill, R.I., Fox, N.C., Ashburner, J., Friston, K.J., Chan, D., Crum, W.R., Rossor, M.N., Frackowiak, R.S., 2002. Automatic differentiation of anatomical patterns in the human brain: validation with studies of degenerative dementias. NeuroImage 17, 29-46.

Hiscock, M., Perachio, N., Inch, R., 2001. Is there a sex difference in human laterality? IV. An exhaustive survey of dual-task interference studies from six neuropsychology journals. J. Clin. Exp. Neuropsychol. 23, $137-148$.

Hutchinson, S., Lee, L.H.L., Gaab, N., Schlaug, G., 2003. Cerebellar volume: gender and musicianship effects. Cereb. Cortex 13, 943-949.

Ifthikharuddin, S.F., Shrier, D.A., Numaguchi, Y., Tang, X., Ning, R., Shibata, D.K., Kurlan, R., 2000. MR volumetric analysis of the human basal ganglia: normative data. Acad. Radiol. 7, 627-634.

Jancke, L., Steinmetz, H., 2003. Anatomical brain asymmetries and their relevance for functional asymmetries. In: Hugdahl, K., Davidson, R.J. (Eds.), The Asymmetrical Brain. The MIT Press, Cambridge, MA, pp. $187-230$.

Jancke, L., Schlaug, G., Steinmetz, H., 1997. Hand skill asymmetry in professional musicians. Brain Cogn. 34, 424-432.

Kansaku, K., Yamaura, A., Kitazawa, S., 2000. Sex differences in lateralization revealed in the posterior language areas. Cereb. Cortex 10, $866-872$. 
Keenan, J.P., Thangaraj, V., Halpern, A.R., Schlaug, G., 2001. Absolute pitch and planum temporale. NeuroImage 14, 1402-1408.

Krams, M., Quinton, R., Ashburner, J., Friston, K.J., Frackowiak, R.S., Bouloux, P.M., Passingham, R.E., 1999. Kallmann's syndrome: mirror movements associated with bilateral corticospinal tract hypertrophy. Neurology 52, 816-822.

Kulynych, J.J., Vladar, K., Jones, D.W., Weinberger, D.R., 1994. Gender differences in the normal lateralization of the supratemporal cortex: MRI surface-rendering morphometry of Heschl's gyrus and the planum temporale. Cereb. Cortex 4, 107-118.

Lake, D.A., Bryden, M.P., 1976. Handedness and sex differences in hemispheric asymmetry. Brain Lang. 3, 266-282.

Lee, D.J., Chen, Y., Schlaug, G., 2003. Corpus callosum: musician and gender effects. NeuroReport 14, 205-209.

LeMay, M., 1977. Asymmetries of the skull and handedness. Phrenology revisited. J. Neurol. Sci. 32, 243-253.

Loftus, W.C., Tramo, M.J., Thomas, C.E., Green, R.L., Nordgren, R.A., Gazzaniga, M.S., 1993. Three-dimensional quantitative analysis of hemispheric asymmetry in the human superior temporal region. Cereb. Cortex 3, 348-355.

Medland, S.E., Geffen, G., McFarland, K., 2002. Lateralization of speech production using verbal/manual dual tasks: meta-analysis of sex differences and practice effects. Neuropsychologia 40, 1233-1239.

Munte, T.F., Altenmuller, E., Jancke, L., 2002. The musician's brain as a model of neuroplasticity. Nat. Rev., Neurosci. 3, 473-478.

Nopoulos, P., Flaum, M., O’Leary, D., Andreasen, N.C., 2000. Sexual dimorphism in the human brain: evaluation of tissue volume, tissue composition and surface anatomy using magnetic resonance imaging. Psychiatry Res. 98, 1-13.

Ohnishi, T., Matsuda, H., Asada, T., Aruga, M., Hirakata, M., Nishikawa, M., Katoh, A., Imabayashi, E., 2001. Functional anatomy of musical perception in musicians. Cereb. Cortex 11, 754-760.

Paus, T., Otaky, N., Caramanos, Z., MacDonald, D., Zijdenbos, A., D’Avirro, D., Gutmans, D., Holmes, C., Tomaiuolo, F., Evans, A.C., 1996. In vivo morphometry of the intrasulcal gray matter in the human cingulate, paracingulate, and superior-rostral sulci: hemispheric asymmetries, gender differences and probability maps. J. Comp. Neurol. 376, $664-673$.

Ratcliff, G., Dila, C., Taylor, L., Milner, B., 1980. The morphological asymmetry of the hemispheres and cerebral dominance for speech: a possible relationship. Brain Lang. 11, 87-98.

Sadato, N., Ibanez, V., Deiber, M.P., Hallett, M., 2000. Gender difference in premotor activity during active tactile discrimination. NeuroImage 11, $532-540$

Schlaug, G., 2001. The brain of musicians. A model for functional and structural adaptation. Ann. N. Y. Acad. Sci. 930, 281-299.

Schlaug, G., Jancke, L., Huang, Y., Staiger, J.F., Steinmetz, H., 1995a. Increased corpus callosum size in musicians. Neuropsychologia 33, $1047-1055$.

Schlaug, G., Jancke, L., Huang, Y., Steinmetz, H., 1995b. In vivo evidence of structural brain asymmetry in musicians. Science 267, 699-701.
Schneider, P., Scherg, M., Dosch, H.G., Specht, H.J., Gutschalk, A., Rupp, A., 2002. Morphology of Heschl's gyrus reflects enhanced activation in the auditory cortex of musicians. Nat. Neurosci. 5, 688-694.

Shahin, A., Bosnyak, D.J., Trainor, L.J., Roberts, L.E., 2003. Enhancement of neuroplastic P2 and N1c auditory evoked potentials in musicians. J. Neurosci. 23, 5545-5552.

Shapleske, J., Rossell, S.L., Woodruff, P.W., David, A.S., 1999. The planum temporale: a systematic, quantitative review of its structural, functional and clinical significance. Brain Res. Brain Res. Rev. 29, $26-49$.

Sluming, V., Barrick, T., Howard, M., Cezayirli, E., Mayes, A., Roberts, N., 2002. Voxel-based morphometry reveals increased gray matter density in Broca's area in male symphony orchestra musicians. NeuroImage $17,1613-1622$.

Sowell, E.R., Thompson, P.M., Holmes, C.J., Batth, R., Jernigan, T.L., Toga, A.W., 1999. Localizing age-related changes in brain structure between childhood and adolescence using statistical parametric mapping. NeuroImage 9, 587-597.

Steinmetz, H., 1996. Structure, functional and cerebral asymmetry: in vivo morphometry of the planum temporale. Neurosci. Biobehav. Rev. 20, $587-591$.

Stewart, L., Henson, R., Kampe, K., Walsh, V., Turner, R., Frith, U., 2003. Brain changes after learning to read and play music. NeuroImage 20, $71-83$.

Tisserand, D.J., Pruessner, J.C., Sanz Arigita, E.J., van Boxtel, M.P., Evans, A.C., Jolles, J., Uylings, H.B., 2002. Regional frontal cortical volumes decrease differentially in aging: an MRI study to compare volumetric approaches and voxel-based morphometry. NeuroImage 17, $657-669$.

Toga, A.W., Thompson, P.M., 2003. Mapping brain asymmetry. Nat. Rev., Neurosci. 4, 37-48.

Wada, J.A., Clarke, R., Hamm, A., 1975. Cerebral hemispheric asymmetry in humans. Cortical speech zones in 100 adults and 100 infant brains. Arch. Neurol. 32, 239-246.

Ward, W.D., Burns, E.M., 1982. Absolute pitch. In: Deutsch, D. (Ed.), The Psychology of Music. Academic Press, New York, pp. 351-431.

Watkins, K.E., Paus, T., Lerch, J.P., Zijdenbos, A., Collins, D.L., Neelin, P., Taylor, J., Worsley, K.J., Evans, A.C., 2001. Structural asymmetries in the human brain: a voxel-based statistical analysis of 142 MRI scans. Cereb. Cortex 11, 868-877.

Woermann, F.G., Free, S.L., Koepp, M.J., Sisodiya, S.M., Duncan, J.S., 1999. Abnormal cerebral structure in juvenile myoclonic epilepsy demonstrated with voxel-based analysis of MRI. Brain 122 (Pt. 11), 2101-2108.

Yeterian, E.H., Pandya, D.N., 1998. Corticostriatal connections of the superior temporal region in rhesus monkeys. J. Comp. Neurol. 399, 384-402.

Zatorre, R.J., Perry, D.W., Beckett, C.A., Westbury, C.F., Evans, A.C., 1998. Functional anatomy of musical processing in listeners with absolute pitch and relative pitch. Proc. Natl. Acad. Sci. U. S. A. 95, 3172-3177. 